\title{
High expression of active ATF6 aggravates endoplasmic reticulum stress-induced vascular endothelial cell apoptosis through the mitochondrial apoptotic pathway
}

\author{
JINGYONG HUANG ${ }^{1,2}$, LI WAN ${ }^{3}$, HEPING LU ${ }^{2}$ and XIAOQIANG LI ${ }^{1}$ \\ ${ }^{1}$ Department of Vascular Surgery, The Second Affiliated Hospital of Soochow University, \\ Soochow, Jiangsu 215000; Departments of ${ }^{2}$ Vascular Surgery and ${ }^{3}$ Pathology, \\ The First Affiliated Hospital of Wenzhou Medical University, Wenzhou, Zhejiang 325000, P.R. China
}

Received March 23, 2017; Accepted November 21, 2017

DOI: $10.3892 / \mathrm{mmr} .2018 .8658$

\begin{abstract}
Activating transcription factor 6 (ATF6), one of three sensor proteins in the endoplasmic reticulum (ER), is an important regulatory factor in the ER stress-induced apoptosis pathway. Although recent studies have made some progress in elucidating the regulation mechanism of ATF6, the specific regulatory mechanism of ER stress-induced vascular endothelial cell (VEC) apoptosis is still unclear. The present study was designed to investigate the role of ATF6 in VECs under thapsigargin (TG)-induced ER stress. ATF6 (1-366aa; ATF6 high-expressed plasmid) and ATF6 (151-366aa; plasmid without transcriptional activity) were transfected into VECs to yield an ATF6 high-expression model and a positive control model, respectively. High expression of ATF6 decreased viability and aggravated ER stress-induced apoptosis in VECs. Increased expression of apoptosis-related genes, including those encoding caspase-3, caspase-9, C/EBP homologous protein (CHOP), cytochrome $c$ and B-cell lymphoma-associated protein $\mathrm{X}(\mathrm{Bax}) / \mathrm{B}$-cell lymphoma (Bcl-)2, was detected by polymerase chain reaction and western blotting in the ATF6 (1-366aa) + TG group. No significant effect of TG treatment and high ATF6 expression was indicated on the expression of death receptor-related genes, including those encoding caspase- 8 and Fas. The results demonstrated that high expression of activated ATF6 aggravates ER stress-induced VEC apoptosis through the mitochondrial apoptotic pathway. Furthermore, in response to ER stress, ATF6 upregulates the expression of caspase-3, caspase-9, CHOP, cytochrome $c$ and $\mathrm{Bax} / \mathrm{Bcl}-2$.
\end{abstract}

Correspondence to: Dr Xiaoqiang Li, Department of Vascular Surgery, The Second Affiliated Hospital of Soochow University, 1055 Sanxiang Road, Soochow, Jiangsu 215000, P.R. China E-mail: xiaoqiang_li233@163.com

Key words: endoplasmic reticulum stress, vascular endothelial cells, activating transcription factor 6 , apoptosis pathway

\section{Introduction}

As a barrier between vessel lumen and surrounding tissues, vascular endothelial cells (VEC) are involved in many aspects of vascular biology, including flow adjustment, substance exchange, prevention of lipid leakage, and inhibition of platelet aggregation. Highly developed endoplasmic reticulum (ER), which realizes secretion function of cells, is observed under electron microscopy in VECs, especially in regenerated vessel endothelium. This presence of the ER results in high sensitivity of VEC to ER induction factors. ER stress is a newly discovered pathway that can initiate the apoptotic process. Many pathophysiological processes and diseases, such as atherosclerosis, diabetes, ischemia/reperfusion and injury, are implicated in ER stress-induced VEC apoptosis (1-3).

The ER is an important organelle in eukaryotes that provides an environment for proteins to synthesize, fold, and assemble, and offers storage sites for $\mathrm{Ca}^{2+}$. The ER is also involved in regulation of $\mathrm{Ca}^{2+}$ balance and metabolism of cholesterol and phospholipids (4,5). Under the presence of induction factors, e.g. oxidative stress, hypoxia, calcium dyshomeostasis, and virus infection, ER stress tends to be induced by the massive accumulation of unfolded and misfolded proteins (6-9). Unfolded protein response (UPR), endoplasmic reticulum-overload response (EOR), and sterol regulatory cascade are three signalling pathways that are activated to help maintain cellular homeostasis. When severe stress is continuous, cell apoptosis processes are eventually induced (10).

UPR is the most important and most clearly studied pathway $(11,12)$. In the early stage of ER stress, through the UPR pathway, misfolded proteins are eliminated and correct protein folding is promoted to recover cellular physiological function and to re-establish ER homeostasis. UPR is mediated by binding immunoglobulin heavy chain protein (BiP)/glucose-regulated protein 78 (GRP78) along with three ER stress sensor proteins: Inositol requiring kinase (IRE), PERK, and activating transcription factor 6 (ATF6) (13). ATF6 is a type 2 transmembrane protein kinase embedded in the ER. In response to ER stress, ATF6 is transported from the ER to the Golgi apparatus and cleaved into a transcriptionally active 
p50ATF6. p50ATF6 then combines with the promoter of ER stress response elements in the nucleus, and induces expression of ER stress-related genes such as those encoding C/EBP homologous protein (CHOP)/growth arrest, DNA damage inducible gene 153 (GADD153), BiP/GRP78, X-box-binding protein 1 (XBP1), and calretinin, which regulate cell survival or apoptosis (14-16).

Nakanishi et al (17) documented that ATF6 regulates ER stress-induced apoptosis of myogenous cells by activating caspase-12. Morishima et al (18) found that ATF6 in rat myoblasts regulate cell apoptosis by specifically suppressing Mcl-1 and up-regulating WBP1. The regulatory pathways of activated ATF6 in different cells are not the same, so the mechanism and pathway in ER stress-induced VEC apoptosis is still unclear. Therefore, the present study used thapsigargin (TG) as an ER stress inducer to investigate the role of ATF6 in VEC apoptosis in response.

\section{Materials and methods}

Recombinant plasmids construction. Recombinant plasmids ATF6 (1-366aa) and ATF5 (151-366aa) were purchased from Shanghai Transheep Biotechnology Co. Ltd., Shanghai, China). ATF6 (1-366aa) was ATF6 high-expressed plasmid, the specific sequences is 5'-CCCAAGCTTATGGGGGGA GCCGGCTGGGGT-3' for sense primer and 5'-ACGCGT CGACGTTCTCTGACACAACTTCAT-3 for reverse primer. ATF6 (151-366aa) was plasmid without transcriptional activity, the specific sequences is 5'-CCCAAGCTTATGGAT AAGCCTGTCACTGGTCC-3' for sense primer and 5'-ACG CGTCGACGTTCTCTGACACAACTTCAT-3' for reverse primer.

Cell infection and treatment. VECs (HUVEC-12 cell line) were purchased from Bogoo Biotechnology Co. Ltd. (Shanghai, China). Cells in logarithmic growth phase were seeded into a 6-well plate and cultured for $24 \mathrm{~h}$. Transfection of recombinant plasmids of ATF6 (1-366aa+) and ATF6 (151-366aa) was performed with Invitrogen Lipofectamine ${ }^{\mathrm{TM}}$ LTX according to the manufacturer's instructions (Thermo Fisher Scientific Inc., New York, NY, USA). Two microgram of Pires2-ZsGreen1-vector or pIRES2-ZsGreen1-ARHGAP18 (Sangon Biotech Inc.,Shanghai,China), $5 \mu$ l of Lipofectamine ${ }^{\mathrm{TM}}$ LTX (Thermo Fisher Scientific Inc.) and $250 \mu$ l Opti-MEM (Shanghai Haoran Biological Technology Co. Ltd., Shanghai, China) were mixed and incubated at room temperature for $25 \mathrm{~min}$. Five hundred microlitre of the mixture was added to a 6-well plate with RPMI 1640 medium (Thermo Fisher Scientific Inc.). Then, after $48 \mathrm{~h}$, the transfected cells were harvested for subsequent experiments. Western blotting was performed to detect the expression of ATF6 to test transfection efficiency.

CCK-8 assay. Cells in TG, ATF6 (151-366aa) + TG and ATF6 (1-366aa) + TG groups were treated with $1 \mu \mathrm{M}$ TG for respectively 12, 24 and $48 \mathrm{~h}$. Cell viability in each group was detected by using CCK8 kit (Shanghai Genomeditech Co.,Ltd., Shanghai, China). Cells were seeded into 96-well plats at amount of $100 \mu \mathrm{l}$ per well, then were incubated at $37^{\circ} \mathrm{C}$ in $5 \% \mathrm{CO}_{2}$ incubator for $4 \mathrm{~h}$. Cells were added by $10 \mu \mathrm{l}$ each well CCK reagent, then incubated at $37^{\circ} \mathrm{C}$ in $5 \% \mathrm{CO}_{2}$ incubator for $1-4 \mathrm{~h}$. The optical density (OD) was observed at $450 \mathrm{~nm}$ by a microplate reader (Bio-Rad Laboratories, Inc., Hercules, CA, USA).

Flow cytometry (FCM). Cells in TG only, ATF6 $(151-366 a a)+$ TG, and ATF6 (1-366aa) + TG groups were treated with $1 \mu \mathrm{M}$ TG for $48 \mathrm{~h}$ to induce ER stress. Cells in these three experimental groups plus the normal control group were seeded into 6 -well plates at a density of $2 \times 10^{4}$ cells/well, and digested and collected with EDTA free trypsin (Beijing Solarbio Technology Co., Ltd., Beijing, China). The cells were then stained with Annexin V-FITC and propidium iodide (Qcbio Science and Technologies Co., Ltd., Shanghai, China), and incubated at room temperature for $15 \mathrm{~min}$ in a dark place. The cultures were then analysed by EPICS XL-MCL flow cytometry (Beckman Coulter, Fullerton, CA, USA) at an excitation wave length of $488 \mathrm{~nm}$ and an emission wavelength of $530 \mathrm{~nm}$. The experiment was run three times, and the apoptosis rate for every group was calculated.

RT-PCR. RT-PCR and SYBR Green I chemistry (Beijing Solarbio Technology Co., Ltd.) were applied to investigate the expression of genes in the study. Cells in each group were seeded into 6 -well plates at a density of $2 \times 10^{4}$ cells/well, the total RNA of cells were extracted with Trizol (Thermo Fisher Scientific Inc.), purity and concentration of the extracted RNA were measured on a UV spectrophotometer (Thermo Fisher Scientific Inc.). cDNA was synthesized by reverse transcription, and fluorescence quantitative detection of the target gene was performed afterwards. $\beta$-actin was applied as the internal control to monitor the RT-PCR efficiency. All $\mathrm{RT}$ reactions were performed in triplicate. The primers were designed by Sangon Biotech Co., Ltd. The specific primer sequences for each gene were listed as the follows: 5'GCT GGAAAGCAGCGCATGAA3' and 5'GCGAGTCGCCTC TACTTCCC 3 ' for CHOP (product: $126 \mathrm{bp}$ ); 5'TGTCACTGC GGGAAGGTCTC3' and 5'AGGTGCACACATCCTTGG CT3' for Cyt C (product: 144 bp); 5'GCCAGCAAACTGGTG CTCAA3' and 5'CCAACCACCCTGGTCTTGGA3' for Bax (product: 126 bp); 5'AGTGGGATGCGGGAGATGTG3' and 5'GGTGGACCACAGGTGGCA3' for Bcl-2 (product: 198 bp); 5'GCCCTGTCCTCCAGGTGAAA3' and 5'CTGGGTCCG GGTGCAGTTTA3' for Fas (product: $187 \mathrm{bp}$ ) and 5'GCCGGG ACCTGACTGACTAC 3 ' and 5'GTCAGGCAGCTCGTAGCT CT3' for $\beta$-actin (product: $188 \mathrm{bp}$ ).

Western blotting. Cells in control, non-transfected ATF6 (151-366aa) and ATF6 (1-366aa) groups were harvested and washed twice with PBS, and protein lysed in ice-cold radioimmunoprecipitation assay buffer (Whiga Technology Co., Ltd., Guangdong, China) with freshly added $0.01 \%$ protease inhibitor phenylmethanesulfonyl fluoride (PMSF) (Sigma-Aldrich Co., LLC, Darmstadt, Germany). The cells were incubated on ice for $30 \mathrm{~min}$ and then centrifuged at $10,000 \mathrm{xg}$ for $5 \mathrm{~min}$ at $4^{\circ} \mathrm{C}$. The supernatant $(20-30 \mu \mathrm{g}$ of protein) was collected, resolved by $10 \%$ sodium dodecyl sulphate polyacrylamide gel electrophoresis (Bio-Rad Laboratories, Inc.), and subsequently transferred to a nitrocellulose membrane via western blotting (Millipore, Shanghai, China). Then the expression of protein cleaved-caspase-3, 

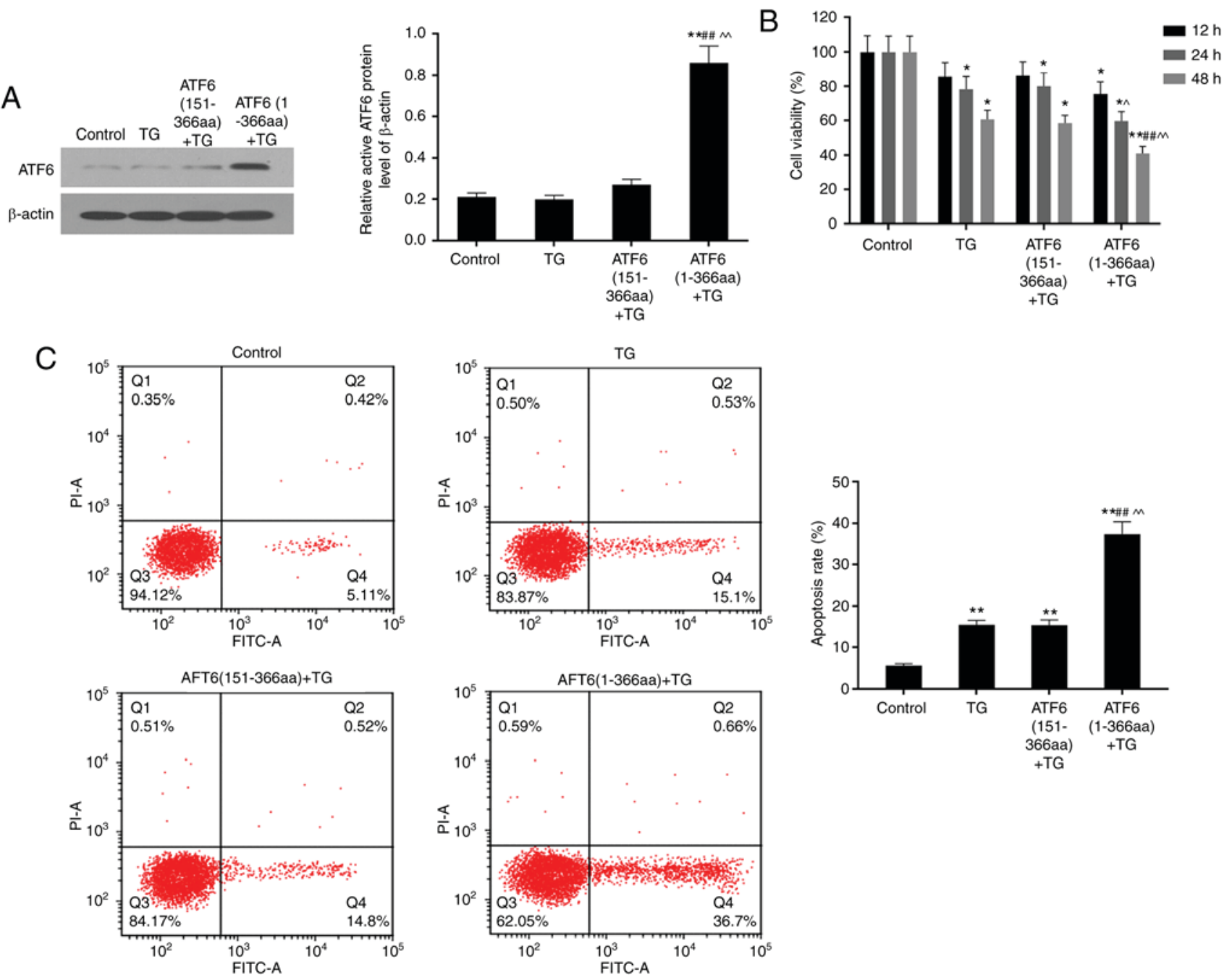

Figure 1. Detection of cell viability and cell apoptosis in control, TG, ATF6 (151-366aa) + TG and ATF6 (1-366aa) + TG groups. (A) High expression of active ATF6 was expressed in cells which were transfected with recombinant ATF6 (1-366aa) plasmid. (B) Treatment with $1 \mu$ M TG was observed to obviously decrease cell viability in time-dependent manner especially in ATF6 (1-366aa) + TG group. (C) High expression of active ATF6 significantly aggravated ER stress induced cell apoptosis. Data were presented as mean $\pm \mathrm{SD}, \mathrm{n}=3,{ }^{*} \mathrm{P}<0.05$ and ${ }^{* *} \mathrm{P}<0.01$ vs. control, ${ }^{\# \#} \mathrm{P}<0.01$ vs. TG treatment $(1 \mu \mathrm{M}$ for $48 \mathrm{~h}),{ }^{\wedge} \mathrm{P}<0.05$ and ${ }^{\wedge} \mathrm{P}<0.01$ vs. ATF6 (151-366aa).

cleaved-caspase-9, cleaved-PARP, cleaved-caspase-4, cleaved-caspase-12, CHOP, cyt c, Bax, Bcl-2, pro-caspase-8, cleaved-caspase-8, Fas, phosphorylated-JNK, JNK and $\mathrm{NF}-\kappa \mathrm{B}$ were detected. Protein loading was estimated using mouse anti- $\beta$-actin monoclonal antibody (Beijing Solarbio Technology Co., Ltd.). Blottings were visualized using enhanced chemiluminescence (Thermo Fisher Scientific Inc.).

Statistical analysis. All values were expressed as mean \pm S.D. Differences between groups were assessed by means of variance analysis and student's t-test. $\mathrm{P}<0.05$ was considered to indicate a statistically significant difference.

\section{Results}

HighexpressionofATF6detectedinATF6(1-366aa)-transfected cells. In testing the transfection efficiency, expression of ATF6 protein was detected through western blotting of ATF6 (151-3aa) and ATF6 (1-366aa) groups. The ATF6 protein was five times more highly expressed in cells that were transfected with the ATF6 (1-366aa) plasmid than in other groups $(\mathrm{P}<0.01)$ (Fig. 1A).

$T G$ treatment for 48 h decreased viability of VEC. Cell viabilities in TG, ATF6 (151-366aa) + TG and ATF6 (1-366aa) + TG groups were inhibited under TG-induced ER stress compared to that inthe control group. ATF6 (1-366aa) + TG cells were the most sensitive to ER stress, showing the lowest viability. Cell viability after TG treatment decreased in a time-dependent manner. Furthermore, there was a significant difference among groups when the cells were treated with TG for $48 \mathrm{~h}$ $(\mathrm{P}<0.01$ or $\mathrm{P}<0.05)$ (Fig. 1B).

TG treatment significantly increased cell apoptosis rate. Compared to that in the control group, cell apoptosis rates in three TG-treated groups increased, particularly in ATF6 (1-366aa)-transfected cells, with the highest significant increase from $5.50 \pm 0.43$ to $37.41 \pm 2.995 \%(\mathrm{P}<0.01)$. Moreover, the apoptosis rate in ATF6 (1-366aa) + TG group was 2.5 times higher than that in the TG only and ATF6 (151-366aa) + TG 

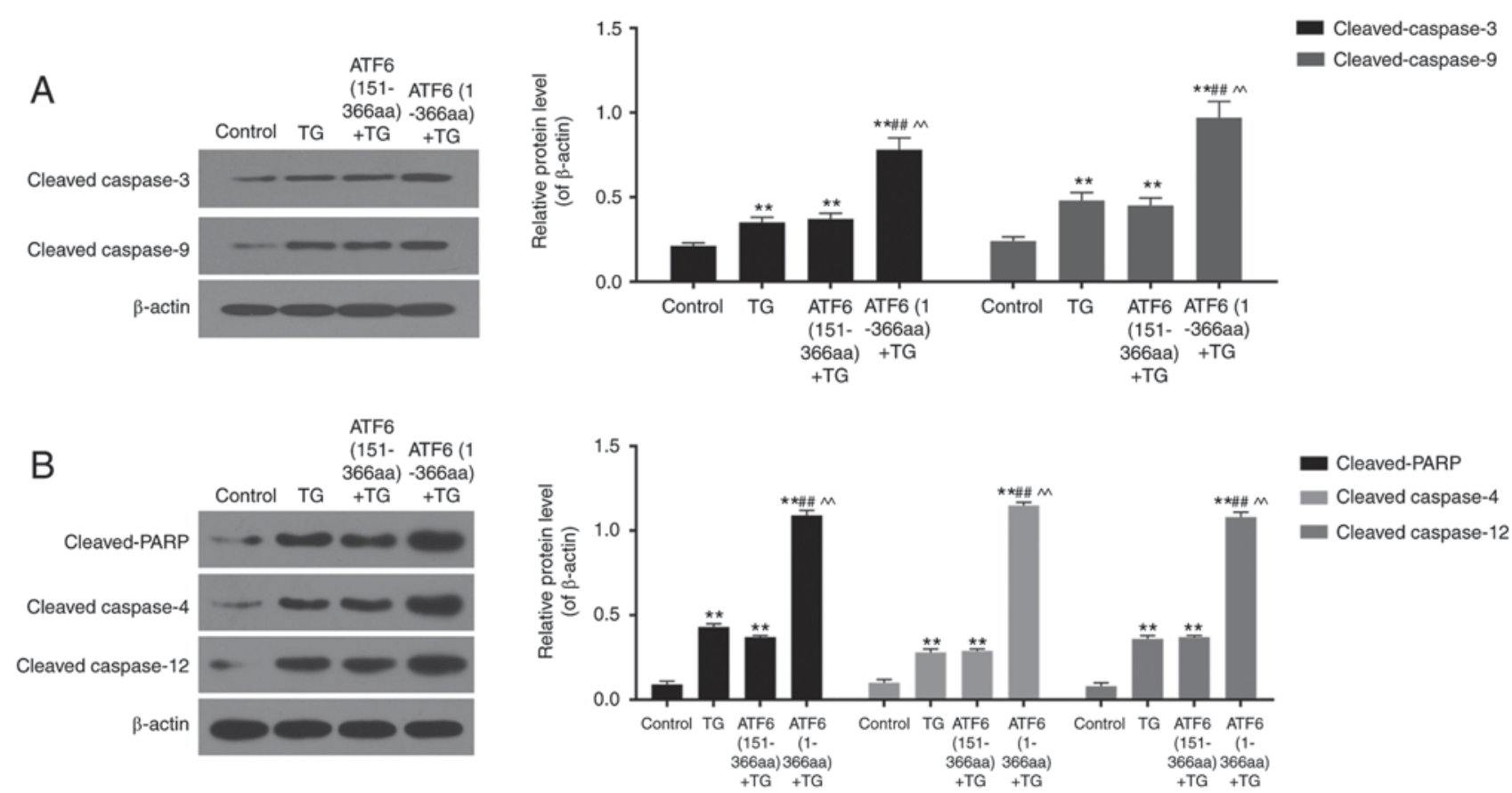

Figure 2. Protein levels of cleaved caspase-3, caspase-9, PARP, caspase-4 and caspase-12 in control, TG, ATF6 (151-366aa) + TG and ATF6 (1-366aa) + TG groups detected using western blotting. (A and B) High expression of active ATF6 up-regulated protein levels of cleaved caspase-3, caspase-9, PARP, caspase- 4 and caspase-12 in ER stress. Data were presented as mean $\pm \mathrm{SD}, \mathrm{n}=3,{ }^{* *} \mathrm{P}<0.01$ vs. control, ${ }^{\# \#} \mathrm{P}<0.01$ vs. TG treatment $(1 \mu \mathrm{M}$ for $48 \mathrm{~h}),{ }^{\wedge} \mathrm{P}<0.01 \mathrm{vs}$. ATF6 (151-366aa).

groups. This reflects that high expression of active ATF6 induces cell apoptosis under ER stress $(\mathrm{P}<0.01)$ (Fig. 1C).

High expression of active ATF6 increased activation of caspase-3, caspase-4, caspase-9 and caspase-12 and cleavage of PARP. TG treatment increased cleavage of caspase-3, caspase-4, caspase-9, caspase-12, and PARP in VEC, when compared to that in the control group $(\mathrm{P}<0.01)$.In ATF6 (1-366aa) + TG group where active ATF6 was over-expressed, the protein levels of cleaved caspase- 3 , caspase- 4 , caspase- 9 , caspase-12, and PARP were significantly higher than those in TG only and ATF6 (151-366aa) + TG groups $(\mathrm{P}<0.01)($ Fig. 2A and B)

High expression of active ATF6 increased expression of CHOP, cyt c, and Bax but led to decreased Bcl-2 protein levels. RT-PCR and western blotting were conducted to analyse the expression levels of apoptosis-related proteins, including CHOP, cyt c, Bax, and Bcl-2. TG treatment of VECs for $48 \mathrm{~h}$ in TG, ATF6 (151-366aa) + TG, and ATF6 $(1-366 a a)+$ TG groups increased mRNA and protein levels of CHOP and cyt $\mathrm{c}$ in comparison with that in the control group $(\mathrm{P}<0.05)$. Expression of these mRNA and proteins in ATF6 (1-366aa) + TG group was significantly different from that in the TG and ATF6 (151-366aa) + TG groups $(\mathrm{P}<0.01)$. Expression levels of Bax mRNA and protein were up-regulated, while those of Bcl-2 were down-regulated by TG treatment $(\mathrm{P}<0.01)$. mRNA expression and protein levels of $\mathrm{CHOP}$ and $\mathrm{Bax}$ were nearly double in ATF6 over-expressing cells, while cyt c levels were approximately three times higher. Up-regulation of ATF6 led to a decreased expression of Bcl-2 (Fig. 3 A-E).

High expression of active ATF6 activated JNK/NF-Kb pathway. The protein levels of total and phosphorylated JNK as well as total NF- $\mathrm{KB}$ were detected by western blotting. The ratio of $\mathrm{p}-\mathrm{JNK} / \mathrm{JNK}$ dramatically increased upon TG treatment $(\mathrm{P}<0.01)$. Phosphorylation levels of JNK was further elevated when active ATF6 was up-regulated in ATF6 (1-366aa) + TG $(\mathrm{P}<0.01)$. High expression of NF- $\kappa \mathrm{B}$ was also observed in TG-treated groups, especially in active ATF6-up-regulated cells where the protein levels of NF- $\kappa \mathrm{B}$ nearly doubled in comparison to that in the TG and ATF6 (151-366aa) + TG groups $(\mathrm{P}<0.01)$ (Fig. 4A).

No significant effect of TG treatment and up-regulation of ATF6 was found on expression of death receptor-related genes. In the present study, Fas mRNA and protein were determined using PCR and western blotting. The results showed no significant difference in its expression among control, TG, ATF6 (151-366aa) + TG and ATF6 (1-366aa) + TG groups ( $\mathrm{P}>0.05)$ (Fig. 4B). Moreover, the protein levels of pro-caspase- 8 and cleaved-caspase- 8 were also found to be not affected by TG treatment and ATF6 expression $(\mathrm{P}>0.05)$ (Fig. 4C).

\section{Discussion}

As VECs are involved in numerous aspects of vascular biology, ER stress-induced VEC apoptosis may be related to numerous pathophysiological processes and diseases (19). As a cell protection mechanism, ER stress reactions reduce protein translation or increase expression of ER BiP to protect cells from ER stress. However, recent research found that if the stress levels are elevated, three pathways including PERK, IRE-1 and ATF6 are activated to induce cell apoptosis (20). ATF6 is one of three sensor proteins in ER stress that induce expression of ER BiP. It was shown that activated ATF6 can 
A
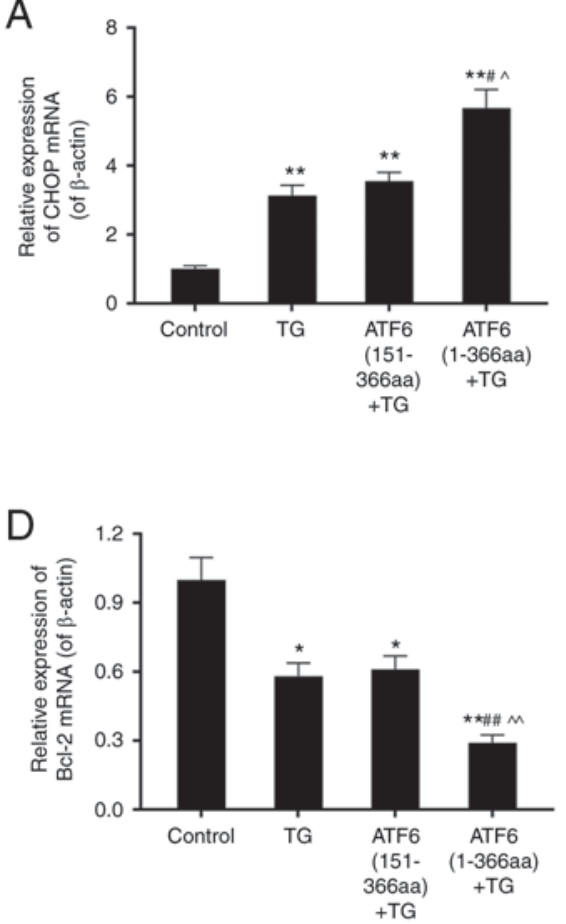

B
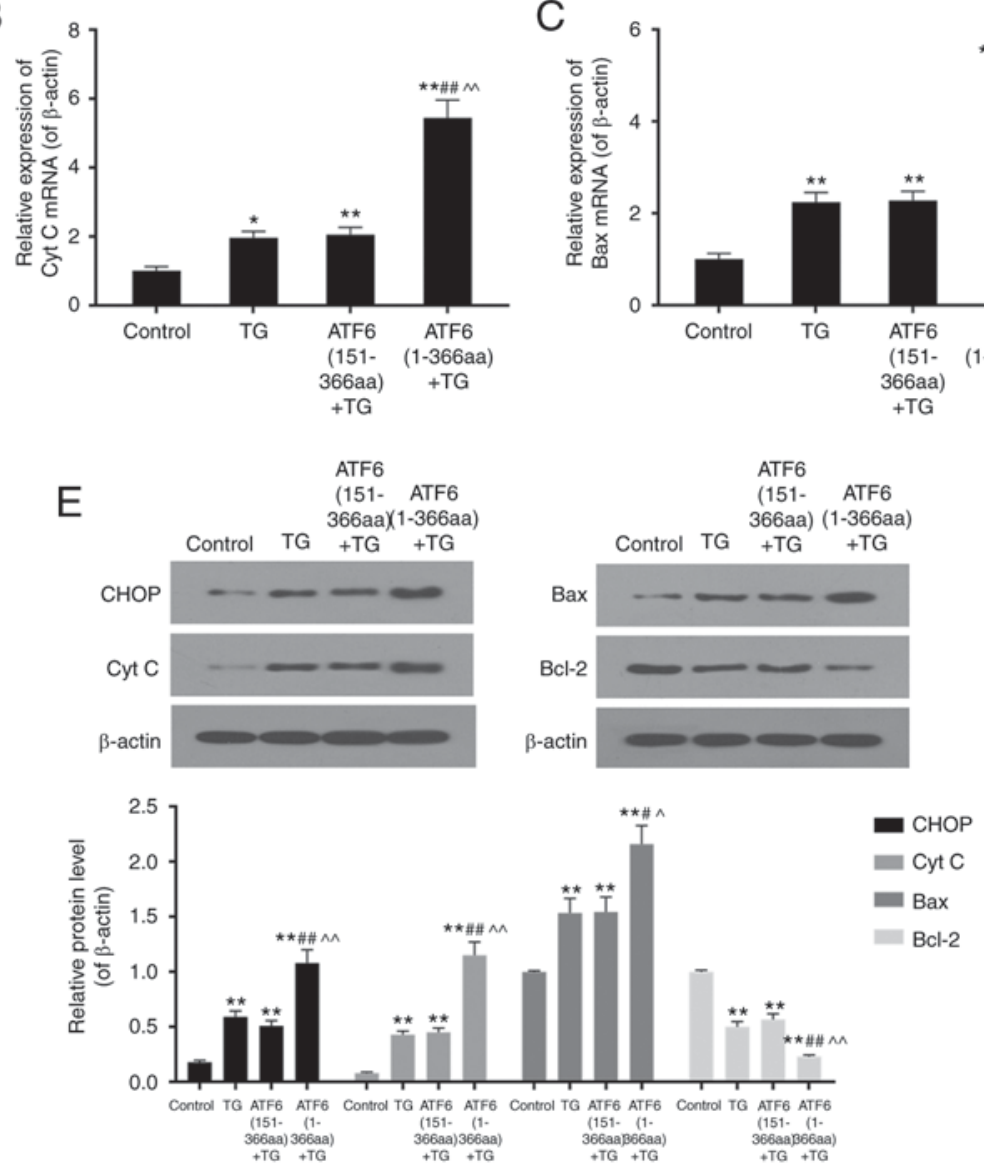

Figure 3. mRNA expression and protein level of apoptosis related genes CHOP, CytC, Bax, and Bcl-2 in control, TG, ATF6 (151-366aa) + TG and ATF6 (1-366aa) + TG groups by means of RT-PCR and western blotting. (A-C) High expression of active ATF6 up-regulated mRNA expression of CHOP, CytC, and Bax in ER stress. (D) High expression of active ATF6 down-regulated mRNA expression of Bcl-2 in ER stress. (E) High expression of active ATF6 up-regulated protein levels of $\mathrm{CHOP}, \mathrm{Cyt} \mathrm{C}$ and $\mathrm{Bax}$, and down regulated that of $\mathrm{Bcl}-2$ in ER stress. Data were presented as mean $\pm \mathrm{SD}$, $\mathrm{n}=3$, ${ }^{*} \mathrm{P}<0.05$ and ${ }^{* * *} \mathrm{P}<0.01$ vs. control, ${ }^{\#} \mathrm{P}<0.05$ and ${ }^{\# \#} \mathrm{P}<0.01$ vs. TG treatment $(1 \mu \mathrm{M}$ for $48 \mathrm{~h}),{ }^{\wedge} \mathrm{P}<0.05$ and ${ }^{\wedge} \mathrm{P}<0.01$ vs. ATF6 (151-366aa).

lead to natural apoptosis of rat myocytes during cell differentiation, which means that activated ATF6 is able to regulate cell apoptosis through activating expression of caspase-12 (17). In rat myoblasts, it is theorised that high expression of activated ATF6 may induce cell apoptosis through down-regulating Mcl-1 and up-regulating WBP1. The pathways of activated ATF6 in regulating cell apoptosis are probably different in different cells (18). Therefore, ATF6 (1-366aa; ATF6 high-expressed plasmid) and ATF6 (151-366aa; plasmid without transcriptional activity) were transfected into VEC to construct ATF6 high-expression model, and TG was applied as an ER stress inducer to explore the role of activated ATF6 in VEC under ER stress.

Apoptosis is a self-destruction process controlled by gene expression, which plays a crucial part in maintenance of homeostasis (21). The mitochondrial pathway and the death receptor-mediated pathway are two classic cell apoptotic pathways that are regulated by caspase- 9 and caspase- 8 , respectively. These pathways finally activate caspase- 3 to induce cell apoptosis (22). For example, when cells are stimulated by drugs, nutrient deficiency, and oxidative stress, caspase- 9 and caspase- 8 are cleaved and activated through diverse pathways, which leads to caspase-3-induced cell apoptosis (23). Recent research reported that ER stress could also initiate apoptotic pathways, although the specific mechanism remained unclear (24).
It is believed that TG, a specific inducer of ER stress, can stimulate ER stress and eventually induce apoptosis in numerous types of cells (25). In the present study, a reduction of VEC viability upon TG treatment was observed, along with a significant increase in the rate of cell apoptosis. In TG-treated VEC, cleaved-caspase-3 and cleaved-caspase-12 levels were both elevated, which implies that TG can induce apoptosis of VEC through ER stress.

Ito et al documented that in TG-treated mouse embryonic fibroblasts, there is an increase in $\mathrm{Ca}^{2+}$ concentration, reduction of mitochondrial membrane potential, release of cyt $\mathrm{c}$ from the mitochondria, cleavage of caspase- 9 and caspase-12, and activation of caspase-3, along with cell apoptosis. This indicates that the mitochondrial pathway is possibly implicated in ER stress-induced cell apoptosis (26). Mitochondria plays a crucial role in the signal transduction process of cell apoptosis, and altering the permeability of the mitochondrial inner membrane is a necessary and sufficient requirement for cell apoptosis (27). In response to external stimuli, permeability of the mitochondrial inner membrane is changed, and pro-caspase- 9 is activated by released cyt c to activate other caspases such as caspase-3, which results in cell apoptosis (28). Our study found that upon TG treatment, cyt c expression was up-regulated with activation of caspase-3, caspase-4, caspase-9, caspase-12, and PARP. Over-expression 

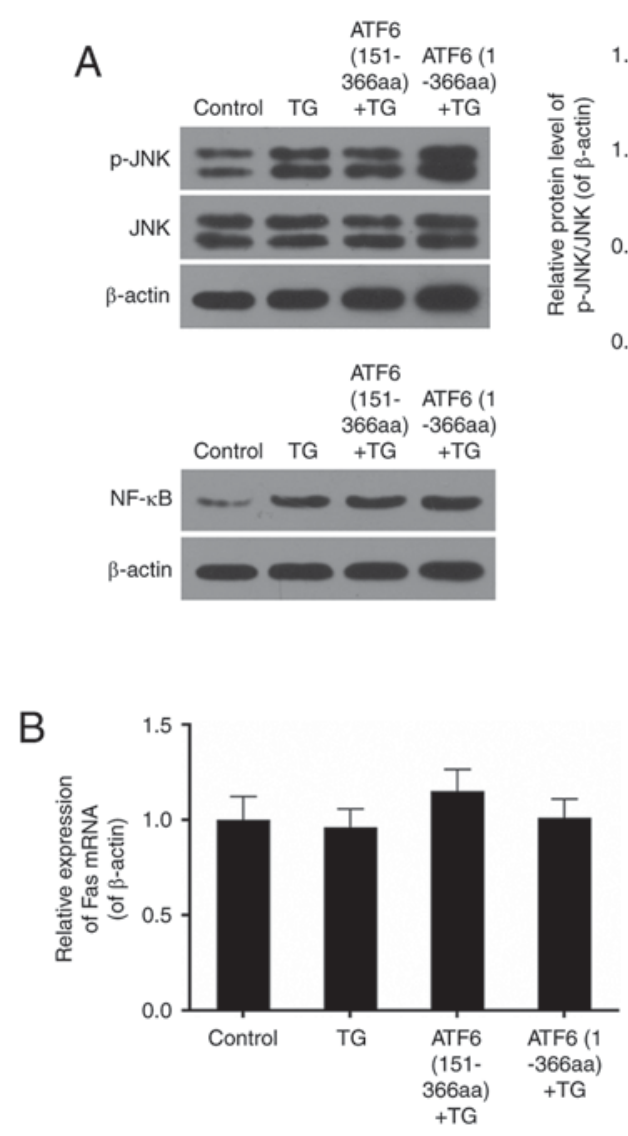
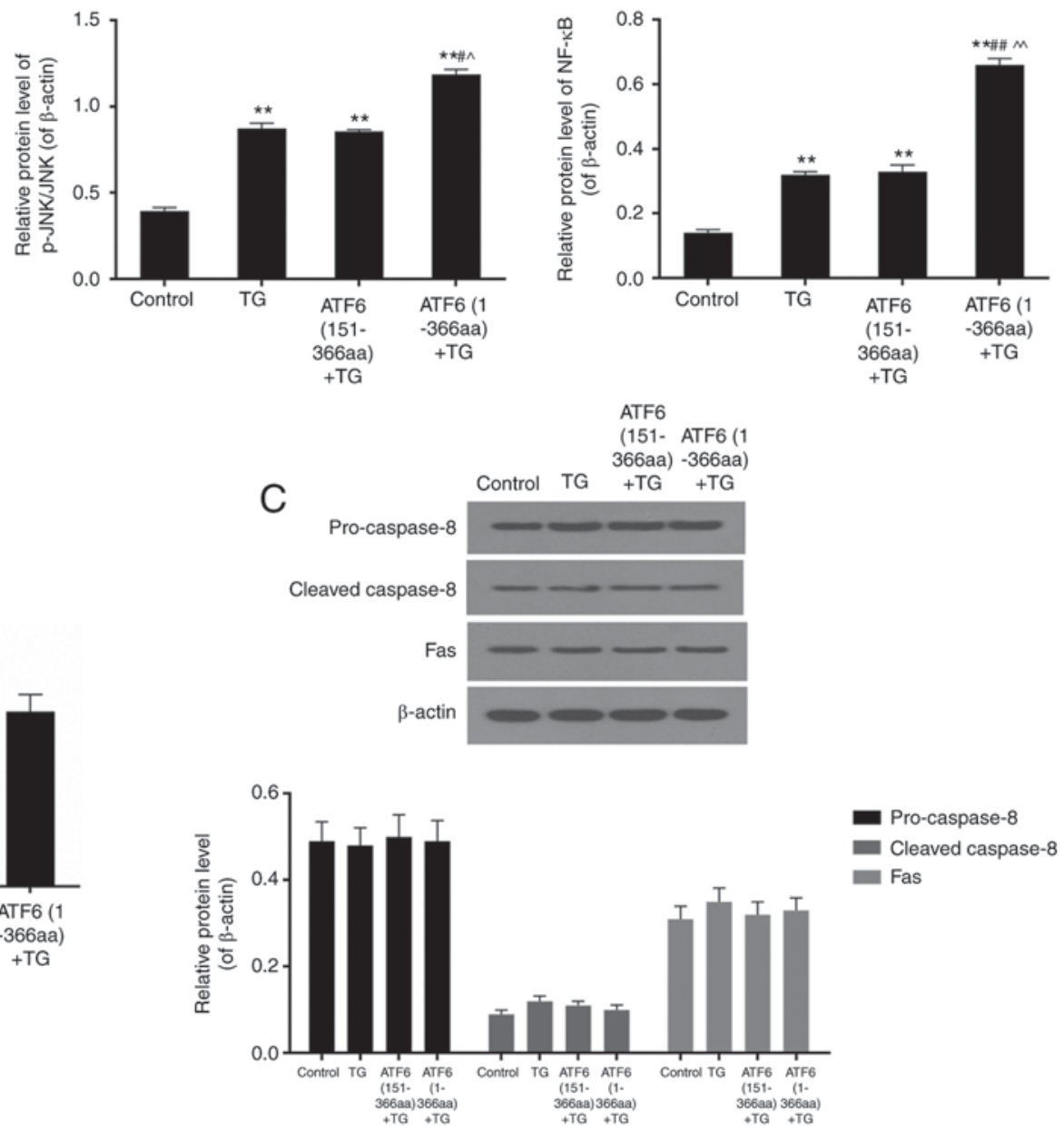

Figure 4. Protein level of JNK/NF-кB, and expression levels of Fas and caspase-8 in control, TG, ATF6 (151-366aa) + TG and ATF6 (1-366aa) + TG groups by means of RT-PCR and western blotting. (A) High expression of active ATF6 activated JNK/NF-kB pathway in ER stress. (B) No significant influence of TG treatment and high expression of active ATF6 on Fas mRNA expression was detected in VEC. (C) No apparent change of protein levels of pro-caspase-8, cleaved-caspase- 8 and Fas was observed in VEC. Data were presented as mean $\pm \mathrm{SD}, \mathrm{n}=3,{ }^{* *} \mathrm{P}<0.01$ vs. control, ${ }^{\#} \mathrm{P}<0.05$ and ${ }^{\# \#} \mathrm{P}<0.01$ vs. TG treatment $(1 \mu \mathrm{M}$ for $48 \mathrm{~h}), \wedge \mathrm{P}<0.05$ and $\wedge \wedge \mathrm{P}<0.01$ vs. ATF6 (151-366aa).

of ATF6 further increased their protein levels as well as that of CHOP. Moreover, activation of JNK/NF- $\mathrm{KB}$ was also detected in ER stress-induced cell apoptosis, especially during ATF6 over-expression, which further supports the role of the mitochondrial pathway.

On the other hand, we found that in VEC with highly expressed and activated ATF6, the expression of mRNA and protein levels of death receptor-related proteins caspase- 8 and Fas was not significantly increased in comparison with that in the control groups. This indicates that the death receptor-mediated pathway may not take part in the ATF6-induced apoptotic process. After the cell was stimulated for apoptosis, cyt c was rapidly released from the mitochondria into the cytoplasm, which is a key event in cell apoptosis (29). In the present study, expression of cyt $\mathrm{c}$ and caspase- 9 was significantly increased in active ATF6 highly expressing cells, which supports the relationship between ATF6 up-regulation and cyt $\mathrm{c}$ release and subsequently, caspase-9 activation.

In mammalian cells, the Bcl-2 family plays an important role in the mitochondrial pathway-mediated cell apoptosis. Different family members play different roles in the apoptotic process. The interaction of anti-apoptotic genes and pro-apoptotic genes is influenced by different levels of reactive oxygen species (ROS), so the relative ratio of pro-apoptotic genes and anti-apoptotic genes of a cell is the key factor in deciding cellular survival or death. At the centre of apoptosis regulation by the Bcl-2 family, lie Bcl-2 and Bax. The expression of Bcl-2 and Bax proteins was used asa marker for apoptosis in the present study $(30,31)$. In tumour cells, many apoptosis-stimulating factors induce the mitochondrial pathway-mediated cell apoptosis through down-regulating the expression of apoptosis inhibitor proteins such as Bcl-2 and $\mathrm{Bcl}-\mathrm{x}_{\mathrm{L}}$, or up-regulating expression of pro-apoptotic proteins like Bax, Bad, and Bid (32). In the present study, we found that the expression of Bax was significantly up-regulated when levels of Bcl-2 were down-regulated in active ATF6 high expressing cells, which indicated that active ATF6-mediated mitochondrial pathway regulates cell apoptosis through down-regulation of the $\mathrm{Bcl}-2 / \mathrm{Bax}$ ratio.

Fas and caspase- 8 are two of main members in death receptor signalling pathway. Transmembrane receptor Fas belongs to tumor necrosis factor super family, and induces apoptosis of numerous types of cells. The death domain in the intracellular region of Fas plays a crucial part in transduction of apoptotic signal. Research has reported that Fas is extensively expressed in immune cells such as activated 
T cells, B cells, natural killer cell and monocyte, and is also found in histocytes, endothelial cells and epithelial cells (33). Fas combines with Fas ligand (FasL), and links with the death domain of Fas-associated death domain protein (FADD) (34). The death effector domain in the n-terminal of FADD peptide chain can gather caspase- 8 zymogen to inform death inducing signalling complex (DISC) with Fas receptor, leading to activation of caspase- 8 and eventually resulting in activation of caspase-3. Activated caspase-3, as the key effector enzyme in apoptosis signal transduction, induces cell apoptosis at the end $(35,36)$. The result of the present study manifested no significant difference in the levels of Fas as well as pro- and cleaved-caspase- 8 among experimental groups. It was implied that ER stress-induced VEC apoptosis is likely not through the death receptor signalling pathway, and ATF6 does not affect the activation of the pathway.

The present study probed into the role of active ATF6 in response to ER stress-induced VEC apoptosis, and demonstrated that high expression of active ATF6 could aggravate VEC apoptosis through mitochondrial apoptotic pathway. The main limitation, however, is no comparison of the influences of high expression of active ATF6 between cells with the coding plasmid but no TG and those which are submitted to stressful condition. Whether ATF6 alone is implicated in the cause of apoptosis in VEC or any other cell type is required to be further studied.

\section{References}

1. Mannarino E and Pirro M: Endothelial injury and repair: A novel theory for atherosclerosis. Angiology 59 (2 Suppl): 69S-72S, 2008.

2. Luchetti F, Crinelli R, Cesarini E, Canonico B, Guid L, Zerbinati C, Di Sario G, Zamai L, Magnani M, Papa S and Iuliano L: Endothelial cells, endoplasmic reticulum stress and oxysterols. Redox Biol 13: 581-587, 2017.

3. Battson ML, Lee DM and Gentile CL: Endoplasmic reticulum stress and the development of endothelial dysfunction. Am J Physiol Heart Circ Physiol 312: H355-H367, 2017.

4. Verkhratsky A and Toescu EC: Endoplasmic reticulum $\mathrm{Ca}(2+)$ homeostasis and neuronal death. J Cell Mol Med 7: 351-361, 2003.

5. Marciniak SJ and Ron D: Endoplasmic reticulum stress signaling in disease. Physiol Rev 86: 1133-1149, 2006.

6. Verras M, Papandreou I, Lim AL and Denko NC: Tumor hypoxia blocks Wnt processing and secretion through the induction of endoplasmic reticulum stress. Mol Cell Biol 28: 7212-7224, 2008.

7. Kammoun HL, Hainault I, Ferré P and Foufelle F: Nutritional related liver disease: Targeting the endoplasmic reticulum stress. Curr Opin Clin Nutr Metab Care 12: 575-582, 2009.

8. Fu S, Yang L, Li P, Hofmann O, Dicker L, Hide W, Lin X, Watkins SM, Ivanov AR and Hotamisligil GS: Aberrant lipid metabolism disrupts calcium homeostasis causing liver endoplasmic reticulum stress in obesity. Nature 473: 528-531, 2011.

9. He S, Yaung J, Kim YH, Barron E, Ryan SJ and Hinton DR: Endoplasmic reticulum stress induced by oxidative stress in retinal pigment epithelial cells. Graefes Arch Clin Exp Ophthalmol 246: 677-683, 2008.

10. Wu J and Kaufman RJ: From acute ER stress to physiological roles of the unfolded protein response. Cell Death Differ 13: 374-384, 2006.

11. Su HL, Liao CL and Lin YL: Japanese encephalitis virus infection initiates endoplasmic reticulum stress and an unfolded protein response. J Virol 76: 4162-4171, 2002.

12. Pahl HL: Signal transduction from the endoplasmic reticulum to the cell nucleus. Physiol Rev 79: 683-701, 1999.

13. Patil $\mathrm{C}$ and Walter $\mathrm{P}$ : Intracellular signaling from the endoplasmic reticulum to the nucleus: The unfolded protein response in yeast and mammals. Curr Opin Cell Biol 13: 349-355, 2001.
14. Ron D and Walter P: Signal integration in the endoplasmic reticulum unfolded protein response. Nat Rev Mol Cell Biol 8: 519-529, 2007.

15. Bertolotti A, Zhang Y, Hendershot LM, Harding HP and Ron D: Dynamic interaction of $\mathrm{BiP}$ and ER stress transducers in the unfolded-protein response. Nat Cell Biol 2: 326-332, 2000.

16. Schroder M and Kaufman RJ: The mammalian unfolded protein response. Annu Rev Biochem 74: 739-789, 2005.

17. Nakanishi K, Sudo T and Morishima N: Endoplasmic reticulum stress signaling transmitted by ATF6 mediates apoptosis during muscle development. J Cell Biol 169: 555-560, 2005.

18. Morishima N, Nakanishi K and Nakano A: Activating transcription factor-6 (ATF6) mediates apoptosis with reduction of myeloid cell leukemia sequence 1 (Mcl-1) protein via induction of WW domain binding protein 1. J Biol Chem 286: 35227-35235, 2011.

19. Toya SP and Malik AB: Role of endothelial injury in disease mechanisms and contribution of progenitor cells in mediating endothelial repair. Immunobiology 217: 569-580, 2012.

20. Szegezdi E, Logue SE, Gorman AM and Samali A: Mediators of endoplasmic reticulum stress-induced apoptosis. EMBO Rep 7: 880-885, 2006

21. Thompson CB: Apoptosis in the pathogenesis and treatment of disease. Science 267: 1456-1462, 1995.

22. Colell A, Ricci JE, Tait S, Milasta S, Maurer U, Bouchier-Hayes L, Fitzgerald P, Guio-Carrion A, Waterhouse NJ, Li CW, et al: GAPDH and autophagy preserve survival after apoptotic cytochrome c release in the absence of caspase activation. Cell 129: 983-997, 2007.

23. Rathmell JC and Kornbluth S: Filling a GAP(DH) in caspase-independent cell death. Cell 129: 861-863, 2007.

24. Momoi T: Conformational diseases and ER stress-mediated cell death: Apoptotic cell death and autophagic cell death. Curr Mol Med 6: 111-118, 2006.

25. Feng XQ, You Y, Xiao J and Zou P: Thapsigargin-induced apoptosis of K562 cells and its mechanism. Zhongguo Shi Yan Xue Ye Xue Za Zhi 14: 25-30, 2006 (In Chinese).

26. Ito Y, Pandey P, Mishra N, Kumar S, Narula N, Kharbanda S, Saxena $S$ and Kufe D: Targeting of the c-Abl tyrosine kinase to mitochondria in endoplasmic reticulum stress-induced apoptosis. Mol Cell Biol 21: 6233-6242, 2001.

27. Rizzuto R, Pinton P, Carrington W, Fay FS, Fogarty KE, Lifshitz LM, Tuft RA and Pozzan T: Close contacts with the endoplasmic reticulum as determinants of mitochondrial $\mathrm{Ca} 2+$ responses. Science 280: 1763-1766, 1998.

28. Adams JM and Cory S: Apoptosomes: Engines for caspase activation. Curr Opin Cell Biol 14: 715-720, 2002.

29. Hengartner MO: Apoptosis. Death cycle and Swiss army knives. Nature 391: 441-442, 1998.

30. Al-Fatlawi AA, Al-Fatlawi AA, Irshad M, Zafaryab M, Rizvi MM and Ahmad A: Rice bran phytic acid induced apoptosis through regulation of $\mathrm{Bcl}-2 / \mathrm{Bax}$ and $\mathrm{p} 53$ genes in $\mathrm{HepG} 2$ human hepatocellular carcinoma cells. Asian Pac J Cancer Prev 15: 3731-3736, 2014.

31. Płonka J, Latocha M, Kuśmierz D and Zielińska A: Expression of proapoptotic BAX and TP53 genes and antiapoptotic BCL-2 gene in MCF-7 and T-47D tumour cell cultures of the mammary gland after a photodynamic therapy with photolon. Adv Clin Exp Med 24: 37-46, 2015.

32. Korsmeyer SJ, Shutter JR, Veis DJ, Merry DE and Oltvai ZN: Bcl-2/Bax: A rheostat that regulates an anti-oxidant pathway and cell death. Semin Cancer Biol 4: 327-332, 1993.

33. Yamana K, Bilim V, Hara N, Kasahara T, Itoi T, Maruyama R, Nishiyama T, Takahashi K and Tomita Y: Prognostic impact of FAS/CD95/APO-1 in urothelial cancers: Decreased expression of Fas is associated with disease progression. Br J Cancer 93: 544-551, 2005

34. Ashkenazi A and Dixit VM: Death receptors: Signaling and modulation. Science 281: 1305-1308, 1998.

35. Scatena R, Bottoni P, Botta G, Martorana GE and Giardina B: The role of mitochondria in pharmacotoxicology: A reevaluation of an old, newly emerging topic. Am J Physiol Cell Physiol 293: C12-C21, 2007.

36. Gehring S, Rottmann S, Menkel AR, Mertsching J, Krippner-Heidenreich A and Lüscher B: Inhibition of proliferation and apoptosis by the transcriptional repressor Mad1. Repression of Fas-induced caspase-8 activation. J Biol Chem 275: 10413-10420, 2000.

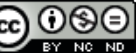

This work is licensed under a Creative Commons Attribution-NonCommercial-NoDerivatives 4.0 International (CC BY-NC-ND 4.0) License. 